\title{
CHOVER-TYPE LAWS OF THE ITERATED LOGARITHM FOR WEIGHTED SUMS OF $\rho^{*}$-MIXING SEQUENCES
}

\author{
GUANG-HUI CAI
}

Received 24 September 2004; Revised 31 May 2005; Accepted 31 May 2005

To derive a Baum-Katz-type result, we establish a Chover-type law of the iterated logarithm for the weighted sums of $\rho^{*}$-mixing and identically distributed random variables with a distribution in the domain of a stable law. Our result obtained not only generalizes the main results of Peng and Qi (2003) and Qi and Cheng (1996) to $\rho^{*}$-mixing sequences of random variables, but also improves them.

Copyright (c) 2006 Guang-hui Cai. This is an open access article distributed under the Creative Commons Attribution License, which permits unrestricted use, distribution, and reproduction in any medium, provided the original work is properly cited.

\section{Introduction}

Let $\left\{X_{i}, i \geq 1\right\}$ be independent and identically distributed (i.i.d.) with symmetric stable distributions, which belong to the domain of normal attraction and nongeneration. So, their characteristic functions are of the forms:

$$
E \exp \left(i t X_{i}\right)=\exp \left(-|t|^{\alpha}\right), \quad t \in R, i \geq 1 \text {. }
$$

Chover [4] has obtained that

$$
\limsup _{n \rightarrow \infty}\left(\frac{\left|\sum_{i=1}^{n} X_{i}\right|}{n^{1 / \alpha}}\right)^{1 / \log \log n}=e^{1 / \alpha} \text { a.s. }
$$

We call this a Chover-type LIL (laws of the iterated logarithm). This type LIL has been established by Vasudeva and Divanji [13], Zinchenko [14] for delayed sums, by Chen and Huang [3] for geometric weighted sums, and by Chen [2] for weighted sums. Qi and Cheng [11] extended the Chover-type law of the iterated logarithm for the partial sums to the case where the underlying distribution is in the domain of attraction of a nonsymmetric stable distribution (see below for details).

Let $L_{\alpha}$ denote a stable distribution with exponent $\alpha \in(0,2)$. Recall that the distribution of $X$ is said to be in the domain of attraction of $L_{\alpha}$ if there exist some constants $A_{n} \in R$ 
2 Chover-type LIL for weighted sums of mixing sequences

and $B_{n}>0$ such that

$$
\frac{S_{n}-A_{n}}{B_{n}} \stackrel{d}{\longrightarrow} L_{\alpha}
$$

Under (1.3), Qi and Cheng [11] and Peng and Qi [10] showed that

$$
\limsup _{n \rightarrow \infty}\left(\frac{\left|\sum_{i=1}^{n} X_{i}-A_{n}\right|}{B_{n}}\right)^{1 / \log \log n}=e^{1 / \alpha} \quad \text { a.s. }
$$

It is well known that (1.3) holds if and only if

$$
1-F(x)=\frac{C_{1}(x) l(x)}{x^{\alpha}}, \quad F(-x)=\frac{C_{2}(x) l(x)}{x^{\alpha}}, \quad \text { for } x>0,
$$

where, for $x>0, C_{i}(x) \geq 0, \lim _{x \rightarrow \infty} C_{i}(x)=C_{i}, i=1,2, C_{1}+C_{2}>0$, and $l(x) \geq 0$ is slowly varying in the sense of Karamata function, that is,

$$
\lim _{t \rightarrow \infty} \frac{l(t x)}{l(t)}=1, \quad \text { for } x>0 .
$$

By Lin et al. [6, page 76, Exercise 21], we have $B_{n}=(n l(n))^{1 / \alpha}$.

For nonempty sets $S, T \subset \mathcal{N}$, we define $\mathscr{F}_{S}=\sigma\left(X_{k}, k \in S\right)$. And we define the maximal correlation coefficient $\rho_{n}^{*}=\sup \operatorname{corr}(f, g)$ where the supremum is taken over all $(S, T)$ with $\operatorname{dist}(S, T) \geq n$ and for all $f \in L_{2}\left(\mathscr{F}_{S}\right), g \in L_{2}\left(\mathscr{F}_{T}\right)$, and $\operatorname{dist}(S, T)=\inf _{x \in S, y \in T}|x-y|$.

A sequence of random variables $\left\{X_{n}, n \geq 1\right\}$ on a probability space $\{\Omega, \mathscr{F}, P\}$ is called $\rho^{*}$-mixing if

$$
\lim _{n \rightarrow \infty} \rho_{n}^{*}=0
$$

As for $\rho^{*}$-mixing sequences of random variables, one can refer to Bryc and Smolenski [1], who established bounds for the moments of partial sums for a sequence of random variables satisfying

$$
\lim _{n \rightarrow \infty} \rho_{n}^{*}<1
$$

Peligrad [7] established a CLT. Peligrad [8] established an invariance principle. Peligrad and Gut [9] established Rosenthal-type maximal inequalities and Baum-Katz-type results. Utev and Peligrad [12] established an invariance principle of nonstationary sequences.

To derive a Baum-Katz-type result, the main purpose of this paper is to establish a Chover-type law of the iterated logarithm for the weighted sums of $\rho^{*}$-mixing and identically distributed random variables with a distribution in the domain of a stable law. Our result not only generalizes the main results of Peng and Qi [10] and Qi and Cheng [11] to $\rho^{*}$-mixing sequences of random variables, but also improves them.

Throughout this paper, let $h \in B[0,1]$ denote that the function $h$ is bounded on $[0,1]$. $C$ will represent a positive constant though its value may change from one appearance to the next, and $a_{n}=O\left(b_{n}\right)$ will mean $a_{n} \leq C b_{n}$. 


\section{The main results}

In order to prove our results, we need the following lemma and definition.

Lemma 2.1 (Utev and Peligrad [12]). Let $\left\{X_{i}, i \geq 1\right\}$ be a $\rho^{*}$-mixing sequence of random variables, $E X_{i}=0, E\left|X_{i}\right|^{p}<\infty$ for some $p \geq 2$ and for every $i \geq 1$. Then there exists $C=C(p)$, such that

$$
E \max _{1 \leq k \leq n}\left|\sum_{i=1}^{k} X_{i}\right|^{p} \leq C\left\{\sum_{i=1}^{n} E\left|X_{i}\right|^{p}+\left(\sum_{i=1}^{n} E X_{i}^{2}\right)^{p / 2}\right\} .
$$

Definition 2.2 ( $\operatorname{Lin}$ and $\mathrm{Lu}[5])$. A function $f(x)>0(x>0)$ is said to be quasimonotone nondecreasing, if

$$
\limsup _{x \rightarrow \infty} \sup _{0 \leq t \leq x} \frac{f(t)}{f(x)}<\infty
$$

Here are our main results.

Theorem 2.3. Let $\left\{X, X_{i}, i \geq 1\right\}$ be a $\rho^{*}$-mixing sequence of identically distributed random variables. Let $h$ be a bounded function on $[0,1]$, continuous at $x_{0} \in(0,1)$. Let $S_{n}=$ $\sum_{i=1}^{n} h(i / n) X_{i}, E X=0$, when $\alpha>1$. Let $f(x)>0$ be quasimonotone nondecreasing and $\int_{1}^{\infty}(1 / x f(x)) d x<\infty$. Then under condition (1.3), for any $\varepsilon>0$,

$$
\sum_{n=1}^{\infty} n^{-1} P\left(\max _{1 \leq j \leq n}\left|S_{j}\right|>\varepsilon(n f(n) l(n))^{1 / \alpha}\right)<\infty .
$$

Proof of Theorem 2.3. For any $i \geq 1$, define $X_{i}^{(n)}=X_{i} I\left(\left|X_{i}\right| \leq a_{n}\right), S_{j}^{(n)}=\sum_{i=1}^{j}\left(h(i / n) X_{i}^{(n)}-\right.$ $\left.E h(i / n) X_{i}^{(n)}\right)$, where $a_{n}=(n f(n) l(n))^{1 / \alpha}$. Then for any $\varepsilon>0$,

$$
\begin{aligned}
& P\left(\max _{1 \leq j \leq n}\left|S_{j}\right|>\varepsilon a_{n}\right) \\
& \quad \leq P\left(\max _{1 \leq j \leq n}\left|X_{j}\right|>a_{n}\right)+P\left(\max _{1 \leq j \leq n}\left|S_{j}^{(n)}\right|>\varepsilon a_{n}-\max _{1 \leq j \leq n}\left|\sum_{i=1}^{j} E h\left(\frac{i}{n}\right) X_{i}^{(n)}\right|\right) .
\end{aligned}
$$

First we show that

$$
\frac{1}{a_{n}} \max _{1 \leq j \leq n}\left|\sum_{i=1}^{j} \operatorname{Eh}\left(\frac{i}{n}\right) X_{i}^{(n)}\right| \longrightarrow 0, \quad \text { as } n \longrightarrow \infty .
$$


4 Chover-type LIL for weighted sums of mixing sequences

In fact, (i) when $0<\alpha \leq 1, h \in B[0,1]$. For any positive integers $n, N$,

$$
\begin{aligned}
\frac{1}{a_{n}} \max _{1 \leq j \leq n}\left|\sum_{i=1}^{j} E h\left(\frac{i}{n}\right) X_{i}^{(n)}\right| \\
\quad \leq \frac{1}{a_{n}} \sum_{i=1}^{n} E\left|h\left(\frac{i}{n}\right) X_{i}^{(n)}\right| \leq \frac{C n}{a_{n}} \int_{|x| \leq a_{n}}|x| d F(x) \\
\quad \leq \frac{C n}{a_{n}} a_{N}+\frac{C n}{a_{n}} \int_{a_{N}<|x| \leq a_{n}}|x| d F(x)=: C(A+B) .
\end{aligned}
$$

Since $f(x)>0$ is a quasimonotone nondecreasing and by (1.5), we have, for $n \geq N, N$ large enough,

$$
\begin{aligned}
B & =\frac{n}{a_{n}} \sum_{k=N+1}^{n} \int_{a_{k-1}<|x| \leq a_{k}}|x| d F(x) \leq \frac{n}{a_{n}} \sum_{k=N+1}^{n} a_{k} P\left(a_{k-1}<|X| \leq a_{k}\right) \\
& \leq C \sum_{k=N+1}^{n} k P\left(a_{k-1}<|X| \leq a_{k}\right) \leq C N P\left(|X| \geq a_{N}\right)+C \sum_{k=N}^{\infty} P\left(|X| \geq a_{k}\right) \\
& \leq C \frac{1}{f(N)}+C \sum_{k=N}^{\infty} \frac{1}{k f(k)} \leq C \frac{1}{f(N)}+C \int_{N}^{\infty} \frac{d x}{k f(k)}<\frac{\varepsilon}{4}
\end{aligned}
$$

It is obvious that for each given $N$,

$$
A \leq C \frac{a_{N}}{(f(n))^{1 / \alpha}} \longrightarrow 0, \quad \text { as } n \longrightarrow \infty .
$$

So, for $0<\alpha \leq 1$, we have (2.5).

(ii) When $1<\alpha<2$, using $E X_{i}=0, h \in B[0,1]$, and (1.5), when $n \rightarrow \infty$, we have

$$
\begin{aligned}
& \frac{1}{a_{n}} \max _{1 \leq j \leq n}\left|\sum_{i=1}^{j} E h\left(\frac{i}{n}\right) X_{i}^{(n)}\right| \\
& \quad=\frac{1}{a_{n}} \max _{1 \leq j \leq n}\left|\sum_{i=1}^{j} E h\left(\frac{i}{n}\right) X_{i} I\left(\left|X_{i}\right|>a_{n}\right)\right| \leq \frac{1}{a_{n}} \sum_{i=1}^{n} E\left|h\left(\frac{i}{n}\right) X_{i}\right| I\left(\left|X_{i}\right|>a_{n}\right) \\
& \quad \leq \frac{C n}{a_{n}} E|X| I\left(|X|>a_{n}\right)=\frac{C n}{a_{n}} \int_{a_{n}}^{\infty} P(|X| \geq x) d x=\frac{C n}{a_{n}} \int_{a_{n}}^{\infty} \frac{C l(n)}{x^{\alpha}} d x \\
& \quad=\frac{n}{a_{n}} C a_{n}^{1-\alpha}=\frac{C}{f(n)}<\frac{\varepsilon}{2} .
\end{aligned}
$$

So, for $1<\alpha<2$, we also have (2.5). Hence (2.5) holds for $0<\alpha<2$.

By (2.4) and (2.5), we have that

$$
P\left(\max _{1 \leq j \leq n}\left|S_{j}\right|>\varepsilon a_{n}\right) \leq \sum_{j=1}^{n} P\left(\left|X_{j}\right|>a_{n}\right)+P\left(\max _{1 \leq j \leq n}\left|S_{j}^{(n)}\right|>\frac{\varepsilon}{2} a_{n}\right),
$$


for $n$ large enough. Hence we need only to prove

$$
\begin{gathered}
I=: \sum_{n=1}^{\infty} n^{-1} \sum_{j=1}^{n} P\left(\left|X_{j}\right|>a_{n}\right)<\infty, \\
I I=: \sum_{n=1}^{\infty} n^{-1} P\left(\max _{1 \leq j \leq n}\left|S_{j}^{(n)}\right|>\frac{\varepsilon}{2} a_{n}\right)<\infty .
\end{gathered}
$$

From (1.5), it is easily seen that

$$
I=\sum_{n=1}^{\infty} P\left(|X|>a_{n}\right) \leq \sum_{n=1}^{\infty} \frac{C}{n f(n)} \leq C \int_{1}^{\infty} \frac{d x}{x f(x)}<\infty .
$$

By Lemma 2.1 and the fact that $h \in B[0,1]$, it follows that

$$
\begin{aligned}
I I & \leq C \sum_{n=1}^{\infty} n^{-1} E \max _{1 \leq j \leq n}\left|S_{j}^{(n)}\right|^{2} \frac{1}{a_{n}^{2}} \leq C \sum_{n=1}^{\infty} n^{-1} \frac{1}{a_{n}^{2}}\left(\sum_{i=1}^{n} E\left|h\left(\frac{i}{n}\right) X_{i}^{(n)}\right|^{2}\right) \\
& \leq C \sum_{n=1}^{\infty} \frac{1}{a_{n}^{2}} E|X|^{2} I\left(|X| \leq a_{n}\right)=C \sum_{n=1}^{\infty} \frac{1}{a_{n}^{2}} \int_{|x| \leq a_{n}} x^{2} d F(x) \\
& =C \sum_{n=1}^{\infty} \frac{1}{a_{n}^{2}} \sum_{k=1}^{n} \int_{a_{k-1}<|x| \leq a_{k}} x^{2} d F(x) \leq C \sum_{k=1}^{\infty} a_{k}^{2} P\left(a_{k-1}<|X| \leq a_{k}\right) \sum_{n=k}^{\infty} \frac{1}{a_{n}^{2}} \\
& \leq C \sum_{k=1}^{\infty} k P\left(a_{k-1}<|X| \leq a_{k}\right) \leq C \int_{1}^{\infty} \frac{d x}{x f(x)}<\infty,
\end{aligned}
$$

which completes the proof of Theorem 2.3.

Corollary 2.4. Under the conditions of Theorem 2.3,

$$
\limsup _{n \rightarrow \infty}\left(\frac{\left|S_{n}\right|}{B_{n}}\right)^{1 / \log \log n} \leq e^{1 / \alpha} \quad \text { a.s. }
$$

Proof of Corollary 2.4. Notice that for any positive integer $n$, there exists an nonnegative integer $k$, such that $2^{k} \leq n<2^{k+1}$. And there exists a $t \in[0,1)$, such that $n=2^{k+t}$. By $(2.3)$, we have

$$
\sum_{k=0}^{\infty} \sum_{n=2^{k}}^{2^{k+1}-1}\left(2^{k+1}-1\right)^{-1} P\left(\max _{1 \leq j \leq 2^{k+t}}\left|S_{j}\right|>\varepsilon\left(2^{k+1} f\left(2^{k+t}\right) l\left(2^{k+t}\right)\right)^{1 / \alpha}\right)<\infty .
$$

Then

$$
\sum_{k=0}^{\infty} P\left(\max _{1 \leq j \leq 2^{k+t}}\left|S_{j}\right|>\varepsilon\left(2^{k+1} f\left(2^{k+t}\right) l\left(2^{k+t}\right)\right)^{1 / \alpha}\right)<\infty .
$$


6 Chover-type LIL for weighted sums of mixing sequences

Then

$$
\frac{\max _{1 \leq j \leq 2^{k+t}}\left|S_{j}\right|}{\left(2^{k+1} f\left(2^{k+t}\right) l\left(2^{k+t}\right)\right)^{1 / \alpha}} \longrightarrow 0 \quad \text { a.s. }
$$

So

$$
\begin{aligned}
\frac{\left|S_{n}\right|}{(n f(n) l(n))^{1 / \alpha}} & \leq \frac{\max _{1 \leq j \leq 2^{k+t}}\left|S_{j}\right|}{\left(2^{k+1} f\left(2^{k+t}\right) l\left(2^{k+t}\right)\right)^{1 / \alpha}} \frac{\left(2^{k+1} f\left(2^{k+t}\right) l\left(2^{k+t}\right)\right)^{1 / \alpha}}{(n f(n))^{1 / \alpha}} \\
& \leq 2^{1 / \alpha} \frac{\max _{1 \leq j \leq 2^{k+t}}\left|S_{j}\right|}{\left(2^{k+1} f\left(2^{k+t}\right)\right)^{1 / \alpha}} \longrightarrow 0 \quad \text { a.s. }
\end{aligned}
$$

Then

$$
\limsup _{n \rightarrow \infty} \frac{\left|S_{n}\right|}{(n f(n) l(n))^{1 / \alpha}}=0 \quad \text { a.s. }
$$

Given $\varepsilon>0$, let $f(x)=\log ^{1+\varepsilon} x$. It is obvious that $\int_{1}^{\infty}(1 / x f(x)) d x<\infty$. By (2.19), we have

$$
\limsup _{n \rightarrow \infty} \frac{\left|S_{n}\right|}{\left(n l(n) \log ^{1+\varepsilon} n\right)^{1 / \alpha}}=0 \quad \text { a.s. }
$$

Then

$$
\limsup _{n \rightarrow \infty}\left(\frac{\left|S_{n}\right|}{B(n)}\right)^{1 / \log \log n} \leq e^{(1+\varepsilon) / \alpha} \quad \text { a.s. }
$$

Therefore

$$
\limsup _{n \rightarrow \infty}\left(\frac{\left|S_{n}\right|}{B(n)}\right)^{1 / \log \log n} \leq e^{1 / \alpha} \quad \text { a.s., }
$$

which completes the proof of (2.14).

Remark 2.5. Corollary 2.4 generalizes the estimate

$$
\limsup _{n \rightarrow \infty}\left(\frac{\left|S_{n}\right|}{B_{n}}\right)^{1 / \log \log n} \leq e^{1 / \alpha} \quad \text { a.s. }
$$

obtained in Peng and Qi [10, Theorem 2.1] to $\rho^{*}$-mixing sequences of random variables. Corollary 2.6. Under the conditions of Corollary 2.4, letting $h(x) \equiv 1$, yields

$$
\limsup _{n \rightarrow \infty}\left(\frac{\left|\sum_{i=1}^{n} X_{i}\right|}{B_{n}}\right)^{1 / \log \log n} \leq e^{1 / \alpha} \quad \text { a.s. }
$$

Remark 2.7. Corollary 2.6 generalizes in Qi and Cheng [11, Theorem 1.1] to $\rho^{*}$-mixing sequences of random variables. 


\section{References}

[1] W. Bryc and W. Smoleński, Moment conditions for almost sure convergence of weakly correlated random variables, Proceedings of the American Mathematical Society 119 (1993), no. 2, 629635.

[2] P. Y. Chen, Limiting behavior of weighted sums with stable distributions, Statistics \& Probability Letters 60 (2002), no. 4, 367-375.

[3] P. Y. Chen and L. H. Huang, The Chover law of the iterated logarithm for random geometric series of stable distribution, Acta Mathematica Sinica 43 (2000), no. 6, 1063-1070 (Chinese).

[4] J. Chover, A law of the iterated logarithm for stable summands, Proceedings of the American Mathematical Society 17 (1966), no. 2, 441-443.

[5] Z. Y. Lin and C. R. Lu, Limit Theory for Mixing Dependent Random Variables, Mathematics and Its Applications, vol. 378, Kluwer Academic, Dordrecht; Science Press, New York, 1996.

[6] Z. Y. Lin, C. R. Lu, and Z. G. Su, Foundation of Probability Limit Theory, Higher Education Press, Beijing, 1999.

[7] M. Peligrad, On the asymptotic normality of sequences of weak dependent random variables, Journal of Theoretical Probability 9 (1996), no. 3, 703-715.

[8] __ Maximum of partial sums and an invariance principle for a class of weak dependent random variables, Proceedings of the American Mathematical Society 126 (1998), no. 4, 11811189.

[9] M. Peligrad and A. Gut, Almost-sure results for a class of dependent random variables, Journal of Theoretical Probability 12 (1999), no. 1, 87-104.

[10] L. Peng and Y. C. Qi, Chover-type laws of the iterated logarithm for weighted sums, Statistics \& Probability Letters 65 (2003), no. 4, 401-410.

[11] Y. C. Qi and P. Cheng, A law of the iterated logarithm for partial sums in the field of attraction of a stable law, Chinese Annals of Mathematics. Series A. Shuxue Niankan. A Ji 17 (1996), no. 2, 195-206 (Chinese).

[12] S. Utev and M. Peligrad, Maximal inequalities and an invariance principle for a class of weakly dependent random variables, Journal of Theoretical Probability 16 (2003), no. 1, 101-115.

[13] K. Vasudeva and G. Divanji, LIL for delayed sums under a non-identically distributed setup, Teoriya Veroyatnosteĭ i ee Primeneniya 37 (1992), no. 3, 534-542 (Russian), translation in Theory Probab. Appl. 37 (1992), no. 3, 497-506.

[14] N. M. Zinchenko, A modified law of iterated logarithm for stable random variables, Theory of Probability and Mathematical Statistics 49 (1994), 69-76 (1995).

Guang-hui Cai: Department of Mathematics and Statistics, Zhejiang Gongshang University, Hangzhou 310035, China

E-mail address: cghzju@163.com 


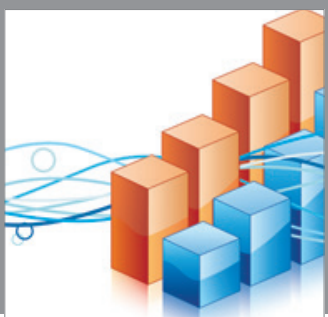

Advances in

Operations Research

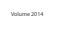

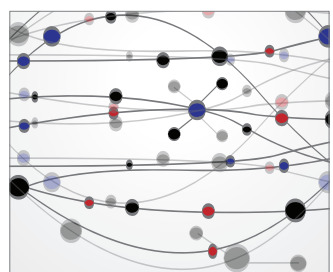

\section{The Scientific} World Journal
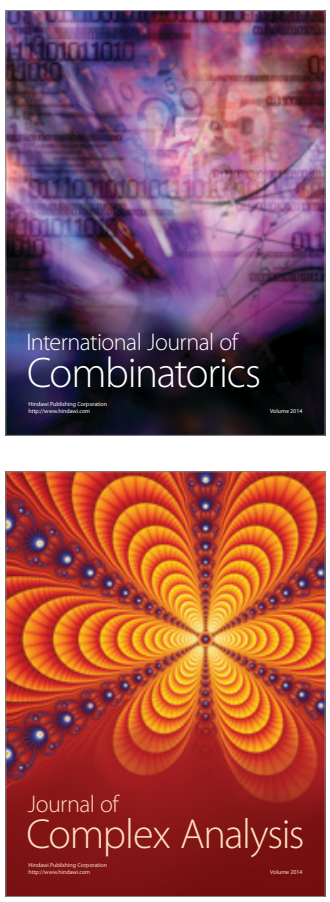

International Journal of

Mathematics and

Mathematical

Sciences
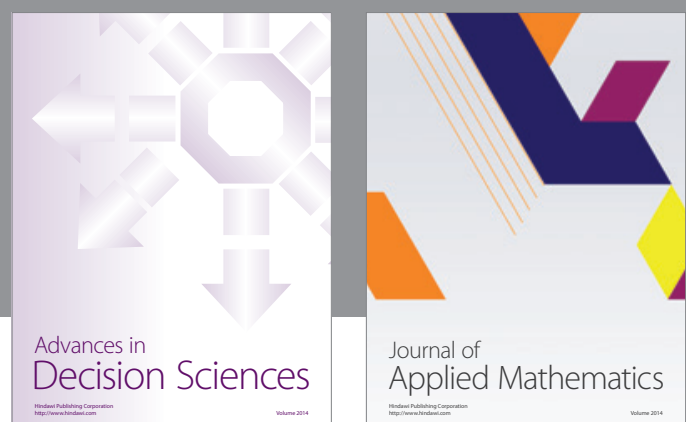

Journal of

Applied Mathematics
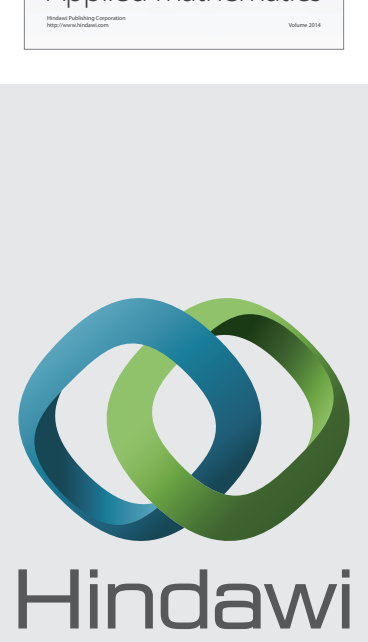

Submit your manuscripts at http://www.hindawi.com
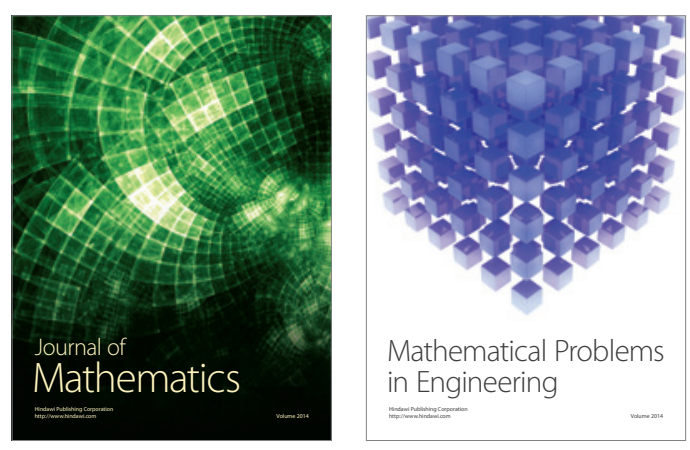

Mathematical Problems in Engineering
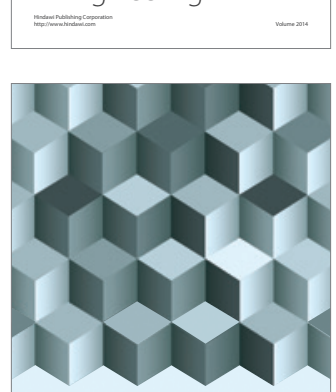

Journal of

Function Spaces
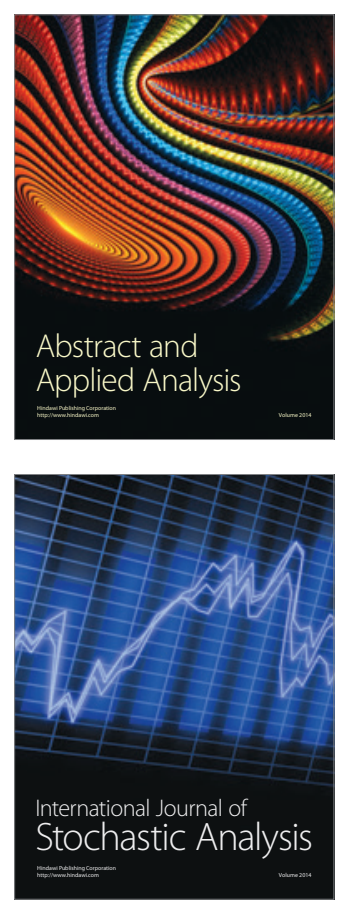

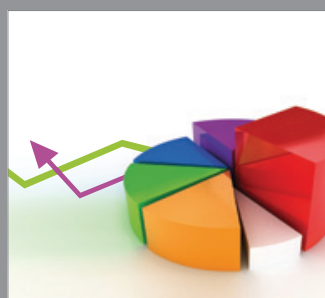

ournal of

Probability and Statistics

Promensencen
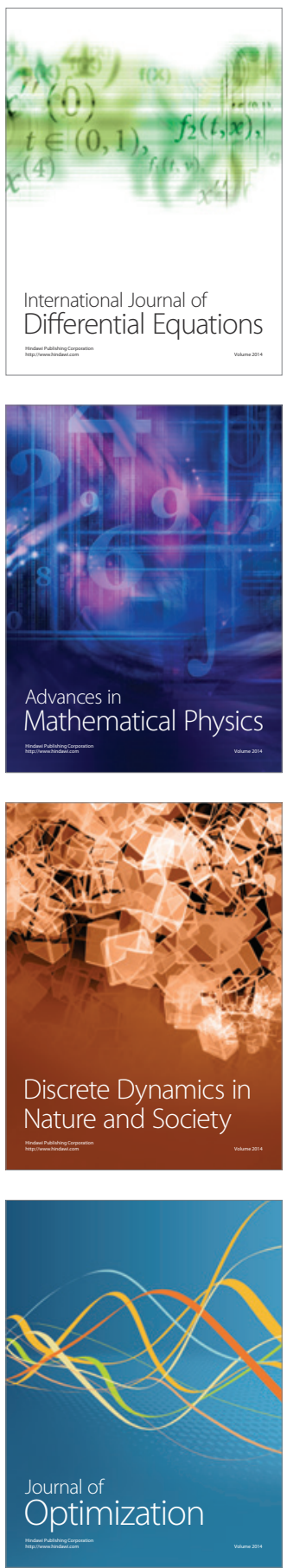